\title{
Collaborative networks enable the rapid establishment of Serological Assays for SARS-CoV-2 during Nationwide Lockdown in New Zealand
}

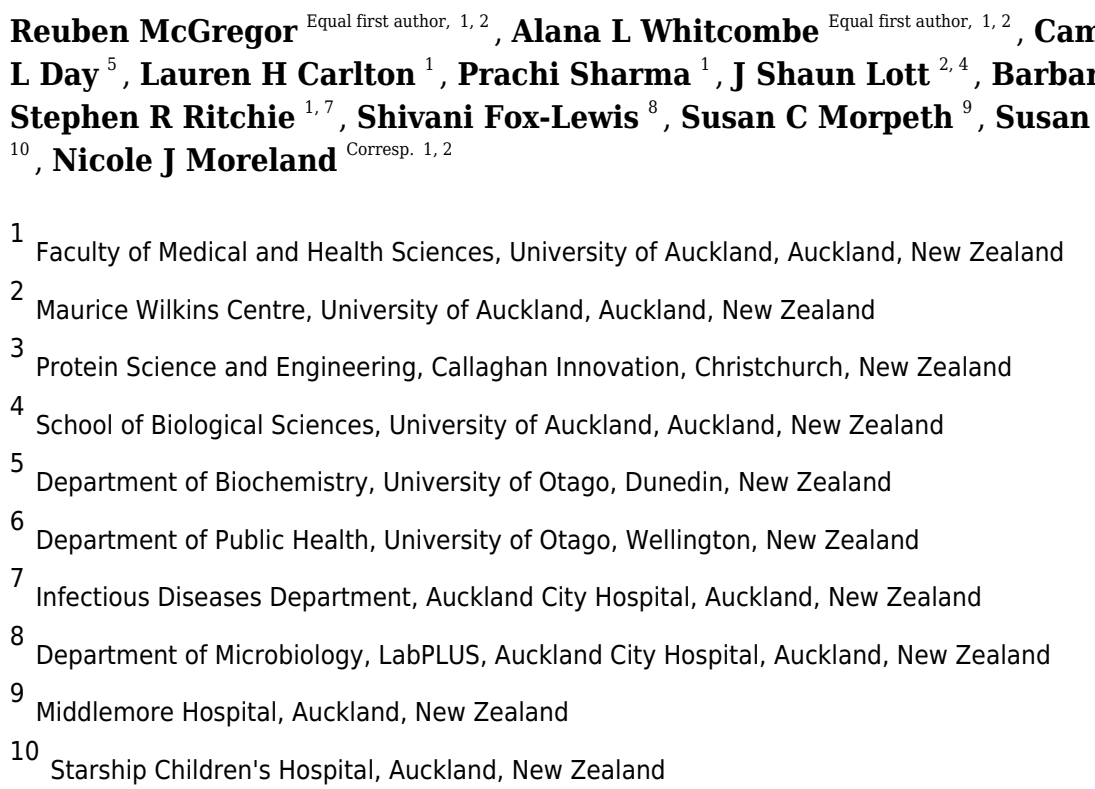

Corresponding Author: Nicole J Moreland

Email address: n.moreland@auckland.ac.nz

Background: Serological assays that detect antibodies to SARS-CoV-2 are critical for determining past infection and investigating immune responses in the COVID-19 pandemic. We established ELISA-based immunoassays using locally produced antigens when NewZealand went into a nationwide lockdown and the supply chain of diagnostic reagents was a widely held domestic concern. The relationship between serum antibody binding measured by ELISA and neutralising capacity was investigated using a surrogate viral neutralisation test (sVNT).

Methods: A pre-pandemic sera panel $(n=113)$, including respiratory infections with symptom overlap with COVID-19, was used to establish assay specificity. Sera from PCR-confirmed SARS-CoV-2 patients $(n=21)$, and PCR-negative patients with respiratory symptoms suggestive of COVID-19 $(n=82)$ that presented to the two largest hospitals in Auckland during the lockdown period were included. A two-step IgG ELISA based on the receptor binding domain (RBD) and Spike protein was adapted to determine seropositivity, and neutralising antibodies that block the RBD/hACE-2 interaction were quantified by sVNT.

Results: The calculated cut-off (>0.2) in the two-step ELISA maximised specificity by classifying all prepandemic samples as negative. Sera from all PCR-confirmed COVID-19 patients were classified as seropositive by ELISA $\geq 7$ days after symptom onset. There was $100 \%$ concordance between the two-step ELISA and the sVNT with all 7+ day sera from PCR-confirmed COVID-19 patients also classified as positive with respect to neutralising antibodies. Of the symptomatic PCR-negative cohort, one individual with notable travel history was classified as positive by two-step ELISA and sVNT, demonstrating the value of serology in detecting prior infection. 
Conclusions: These serological assays were established and assessed at a time when human activity was severely restricted in New Zealand. This was achieved by generous sharing of reagents and technical expertise by the international scientific community, and highly collaborative efforts of scientists and clinicians across the country. The assays have immediate utility in supporting clinical diagnostics, understanding transmission in high-risk cohorts and underpinning longer-term "exit" strategies based on effective vaccines and therapeutics. 
1 Collaborative networks enable the rapid establishment of Serological Assays

2 for SARS-CoV-2 during Nationwide Lockdown in New Zealand

3

4
Reuben McGregor ${ }^{1,2 *}$, Alana L. Whitcombe ${ }^{1,2 *}$, Campbell R. Sheen ${ }^{3}$, James M.J. Dickson ${ }^{4}$, Catherine L. Day ${ }^{5}$, Lauren H. Carlton ${ }^{1,2}$, Prachi Sharma ${ }^{1}$, J. Shaun Lott ${ }^{2,4}$, Barbara Koch ${ }^{3}$, Julie Bennett ${ }^{6}$, Michael G. Baker 6 , Stephen R. Ritchie ${ }^{1,7}$ Shivani Fox-Lewis ${ }^{8}$, Susan C. Morpeth ${ }^{9}$, Susan L. Taylor ${ }^{9}$, Sally A. Roberts ${ }^{2,8}$, Rachel H. Webb ${ }^{1,10}$, Nicole J. Moreland ${ }^{1,2, \#}$

1. Faculty of Medical and Health Sciences, University of Auckland

2. Maurice Wilkins Centre, University of Auckland

3. Protein Science and Engineering, Callaghan Innovation, Christchurch

4. School of Biological Sciences, University of Auckland

5. Department of Biochemistry, University of Otago, Dunedin

6. Department of Public Health, University of Otago, Wellington

7. Infectious Diseases Department, Auckland City Hospital, Auckland

8. Department of Microbiology, LabPLUS, Auckland City Hospital, Auckland

9. Middlemore Hospital, Auckland

10. Starship Children's Hospital and Kidz First Children's Hospital, Auckland

*Equally contributed

\section{\#Corresponding Author:}

Dr Nikki Moreland, Department of Molecular Medicine and Pathology, Faculty of Medical and Health Sciences, University of Auckland, Private Bag 92019, Auckland, New Zealand.

Email: $\underline{\text { n.moreland@auckland.ac.nz }}$ 


\section{ABSTRACT}

29 Background: Serological assays that detect antibodies to SARS-CoV-2 are critical for 30 determining past infection and investigating immune responses in the COVID-19 pandemic. We 31 established ELISA-based immunoassays using locally produced antigens when New Zealand 32 went into a nationwide lockdown and the supply chain of diagnostic reagents was a widely held 33 domestic concern. The relationship between serum antibody binding measured by ELISA and 34 neutralising capacity was investigated using a surrogate viral neutralisation test (sVNT).

35 Methods: A pre-pandemic sera panel $(n=113)$, including respiratory infections with symptom 36 overlap with COVID-19, was used to establish assay specificity. Sera from PCR-confirmed 37 SARS-CoV-2 patients $(\mathrm{n}=21)$, and PCR-negative patients with respiratory symptoms suggestive 38 of COVID-19 $(n=82)$ that presented to the two largest hospitals in Auckland during the

40 41 lockdown period were included. A two-step IgG ELISA based on the receptor binding domain (RBD) and Spike protein was adapted to determine seropositivity, and neutralising antibodies that block the RBD/hACE-2 interaction were quantified by sVNT.

Results: The calculated cut-off $(>0.2)$ in the two-step ELISA maximised specificity by classifying all pre-pandemic samples as negative. Sera from all PCR-confirmed COVID-19 patients were classified as seropositive by ELISA $\geq 7$ days after symptom onset. There was $100 \%$ concordance between the two-step ELISA and the sVNT with all 7+ day sera from PCR-confirmed COVID-19 patients also classified as positive with respect to neutralising antibodies. Of the symptomatic PCR-negative cohort, one individual with notable travel history was classified as positive by two-step ELISA and sVNT, demonstrating the value of serology in detecting prior infection.

Conclusions: These serological assays were established and assessed at a time when human activity was severely restricted in New Zealand. This was achieved by generous sharing of reagents and technical expertise by the international scientific community, and highly collaborative efforts of scientists and clinicians across the country. The assays have immediate utility in supporting clinical diagnostics, understanding transmission in high-risk cohorts and underpinning longer-term "exit" strategies based on effective vaccines and therapeutics. 


\section{INTRODUCTION}

59

60 Severe acute respiratory syndrome coronavirus 2 (SARS-CoV-2), the causative agent of the

61 Coronavirus Disease 2019 (COVID-19) global pandemic, is typically detected in acutely infected

62 individuals via nucleic acid-based polymerase chain reaction (PCR) tests (Sethuraman, Jeremiah

$63 \&$ Ryo, 2020). The first evidence of community transmission in New Zealand was reported on

$6423^{\text {rd }}$ March 2020, the country went into an intense "Alert Level 4" lockdown (the highest level of

65 a 4-level response system) three days later and remained at this Alert Level for the following five

66

67

68

69

70

71

72

73

74

75

76

77

78

79

80

81

82

83

84

85

86

87

88 weeks (Baker, Kvalsvig \& Verrall, 2020). During this time there was notable increase in national nucleic acid testing capacity and this remains the cornerstone of SARS-CoV-2 diagnosis. However, there is also a need for reliable serological assays that measure antibody responses to the virus. While serological assays are not suited to the diagnosis of acute infections due to the days required to generate an antibody response, they are critical for determining past exposure and investigating immune responses (Abbasi, 2020; Sethuraman, Jeremiah \& Ryo, 2020; Krammer \& Simon, 2020).

Numerous laboratory-based serological assays for SARS-CoV-2 are being developed worldwide including enzyme-linked immunosorbent assays (ELISAs) and bead-based immunoassays for measurement of SARS-CoV-2 antibodies (Petherick, 2020; Garcia-Basteiro et al., 2020;

Krammer \& Simon, 2020), as well as virus neutralisation assays for quantification of neutralising antibodies (Tan et al., 2020; Anderson et al., 2020). The spike protein (S protein) expressed by SARS-CoV-2 contains a receptor binding domain (RBD), which interacts with host cells via the human angiotensin-converting enzyme 2 (hACE2) (Diamond \& Pierson, 2020). The S protein is highly immunogenic and, given the integral role of the S protein and RBD in facilitating viral entry, these antigens form the basis of many immunoassays described to date (Duan et al., 2020; Garcia-Basteiro et al., 2020; Long et al., 2020; Amanat et al., 2020). Recent data from immunoassays based on the SARS-CoV-2 nucleocapsid protein also show high sensitivity (Sethuraman, Jeremiah \& Ryo, 2020; Bryan et al., 2020). However, the higher sequence conservation of the SARS-CoV-2 nucleocapsid with other coronaviruses, compared to the S protein, increases the possibility of antibody cross-reactivity against the nucleoprotein in those infected with related viruses (Krammer \& Simon, 2020; Anderson et al., 2020). 
89

90 As New Zealand entered Alert Level 4 Lockdown, and the supply chain of diagnostic reagents

91 for managing COVID-19 testing was a widely held domestic concern, we sought to establish a

92 serologic ELISA assay based on locally-produced SARS-CoV-2 antigens. We adapted the

93 two-step ELISA protocols developed at The Icahn School of Medicine at Mount Sinai (New

94 York City) based on the S protein and RBD, which has FDA Emergency Use Authorization

95 (Amanat et al., 2020; Stadlbauer et al., 2020). A panel of sera/plasma from PCR-confirmed

96 COVID-19 patients was compared with pre-pandemic sera to determine assay parameters. The

97 relationship between antibody binding to the S protein and RBD, and neutralising ability was

98 explored using the surrogate viral neutralisation test (sVNT) recently developed at Duke-NUS

99 (Singapore) (Tan et al., 2020). Finally, the utility of both the ELISA and sVNT to identify prior

100 SARS-CoV-2 infection was investigated in a cohort of PCR-negative patients that presented to

101 hospital with respiratory symptoms during the lockdown period.

102

103

\section{METHODS}

104

\section{Human samples}

106 Human plasma and sera were obtained from several different sources, all of which were granted

107 ethical approval by the University of Auckland Human Ethics Committee or the Health and

108 Disability Ethics Committee in New Zealand. A total of 113 samples collected before 2020 were

109 used as negative controls and all participants (or their parents or legal

110109 guardians) provided written informed consent. These included healthy adult volunteers

111 ( $\mathrm{n}=31)$ (ethics UOA021200), hospitalised adults with bacteraemia or bacterial pneumonia $(\mathrm{n}=25)$

112 (ethics HDEC 17/STH/233), together with children infected with various respiratory viruses

113 ( $\mathrm{n}=57)$ (ethics HDEC 17/NTA/262) (Bennett et al., 2019). The patients with bacterial pneumonia

114 had signs, symptoms and radiological imaging diagnostic of pneumonia and Streptococcus

115 pneumoniae identified. The COVID-19 panel comprised serum and plasma $(\mathrm{n}=21)$ obtained from

11617 patients with PCR-confirmed SARS-CoV-2 infection and those with respiratory symptoms

117 fitting the case definition for COVID-19 testing that were subsequently found negative by PCR

118 ( $\mathrm{n}=82)$. The PCR-confirmed and PCR-negative patients were admitted to Middlemore or

119 Auckland City hospitals in Auckland, New Zealand between March and May 2020 with residual

Peer) reviewing PDF | (2020:07:50726:1:1:NEW 10 Aug 2020) 
120 sera/plasma stored following completion of all routine testing for validation of antibody

121 diagnostics (ethics HDEC 20NTB76) (Table 1). All serum and plasma samples were heated at

$12256^{\circ} \mathrm{C}$ for $30 \mathrm{~min}$ before use to inactivate any residual virus, as published (Amanat et al., 2020).

123 Pooled human intravenous immunoglobulin (IVIG) were produced before 2020 from $>1500$

124 donors in New Zealand (Intragram P) and Europe and North America (Privigen) (CSL Behring).

125

\section{Indirect ELISA}

127 The S protein and RBD antigens were expressed and purified from pCAGGS-RBD and

128 pCAGGS-solSpike vectors respectively, kindly provided by Florian Krammer (The Icahn School

129 of Medicine at Mt Sinai, New York City) using Expi293F or Freestyle293 human embryonic

130 kidney (HEK) cells and published protocols (Stadlbauer et al., 2020), but with a modified

131 transient transfection protocol using polyethyleneimine (PEI). Plasmid DNA was added at 3.5

$132 \mu \mathrm{g} / \mathrm{mL}$ with PEI 7.0 at $\mu \mathrm{g} / \mathrm{mL}$ for $24 \mathrm{hrs}$, after which culture volumes were doubled and

133 supplemented with $2.2 \mathrm{mM}$ valproic acid. Cultures were incubated with shaking for a further 72

134 hours before protein purification was performed.

135

136 The two-step ELISA protocol that includes a single point screen against the RBD, followed by a

137 confirmatory titration against the S protein (Stadlbauer et al., 2020), was utilised with minor

138 modifications. In step one immunoplates were coated with RBD $(5 \mu \mathrm{g} / \mathrm{ml})$ overnight at $4^{\circ} \mathrm{C}$ and

139 blocked with phosphate-buffered saline supplemented with 0.1\% Tween 20 (PBST) and 3\% skim

140 milk powder at $20^{\circ} \mathrm{C}$ for $1 \mathrm{hr}$. Serum or plasma diluted 1:100 in diluent buffer (PBST $+1 \%$ skim

141 milk powder) was added for $1 \mathrm{hr}$ at $20^{\circ} \mathrm{C}$. Following washing (3x PBST), peroxidase-labelled

142 anti-human $\operatorname{IgG}\left(\right.$ Abcam, 97221) diluted 1:10,000 was added for $1 \mathrm{hr}$ at $20^{\circ} \mathrm{C}$. The reaction was

143 developed with 3,3',5,5'-Tetramethylbenzidine (TMB) and stopped with $1 \mathrm{M} \mathrm{HCl}$. The optical

144 density (OD) at 450-570nm was measured using an EnSight absorbance reader. In step two,

145 immunoplates were coated with S protein $(5 \mu \mathrm{g} / \mathrm{ml})$ overnight at $4^{\circ} \mathrm{C}$ and the ELISA was

146 performed using the same protocol as in step one, except that 3-fold serial dilutions of samples

147 starting at 1:100, were prepared. Samples were classified as seropositive if they had an OD

148 above the calculated cut-off $(>0.2)$ in the single point RBD ELISA and in at least two

149 consecutive wells in the $\mathrm{S}$ protein titration ELISA. Positive and negative quality controls were 
150 included on each plate, with the assay meeting acceptance criteria if the OD was $>0.75$ and

$151<0.03$ for the positive and negative control, respectively.

152

153 To assess healthy control IgG reactivity to human coronaviruses (HCoV) ELISA were performed 154 as described above with S1 antigens from the HKU1, NL63 and 229E strain (Sino Biological) 155 coated at $5 \mu \mathrm{g} / \mathrm{ml}$ and a 1:300 sera dilution. Samples from PCR-confirmed COVID-19 patients 156 were also subject to isotype-specific titration ELISA using peroxidase-labelled anti-human IgG 157 (Abcam, 97221), anti-human IgM (Abcam, 97205) and anti-human IgA (Abcam, 97215) at 158 1:10,000 dilution. The area under the curve (AUC) was used to compare isotype-specific 159 antibody titres and was calculated by subtracting background AUC of pooled negative control 160 sera for each isotype in Prism 8 (GraphPad).

161

\section{Surrogate Viral Neutralisation Test (sVNT)}

163 Surrogate neutralization assays were carried out using a SARS-CoV-2 sVNT Kit supplied 164 pre-launch by GenScript as described (Tan et al., 2020). Briefly, serum or plasma was diluted 165 1:20 before incubation with an equivalent volume of peroxidase-conjugated RBD for 30 minutes 166 at $37^{\circ} \mathrm{C}$. This was added to wells pre-coated with human ACE-2 receptor protein and incubated 167 for a further 15 minutes at $37^{\circ} \mathrm{C}$. Following washing and TMB development the OD $450 \mathrm{~nm}$ was 168 measured using an EnSight absorbance reader. Inhibition was calculated as ( 1 - OD sample / OD 169 of negative control) $x 100$. Samples with a percentage inhibition $\geq 20 \%$ were deemed to have 170 neutralizing antibodies.

171

\section{Statistical analysis}

173 The two-step ELISA and sVNT analysis utilized a Kruskal-Wallis test for comparison between 174 three groups with Dunn's multiple comparisons test. AUC data were $\log 10$ transformed to 175 achieve a Gaussian distribution and analysed by one-way ANOVA followed by Tukey's multiple 176 comparisons test. Correlations were calculated using Pearson's correlation coefficient. Data were 177 analysed using Prism 8 (GraphPad) or R (version 3.6.3) within R Studio (version 1.2.5033) and a $178 P$-value of $\leq 0.05$ was considered statistically significant.

179

180

\section{RESULTS}


181

\section{Indirect ELISA with the RDB and S protein}

183 The RBD and S proteins used as antigens in the ELISA were shown to be $>95 \%$ pure by

184 SDS-PAGE following expression and purification from mammalian (HEK derived) cells. In line

185 with previous reports the yield of RBD was approximately 10-fold higher per litre of culture than

186 the S protein (Amanat et al., 2020). To establish ELISA cut-off values a panel of 113 sera

187 collected prior to 2020 were tested, with the cut-off defined as mean plus three-standard

188 deviations. Importantly, this panel included samples from participants with bacterial pneumonia

189 and common respiratory viruses that have symptom overlap with SARS-CoV-2 infections (Table

190 1). The healthy control sera $(n=31)$ within the panel showed broad reactivity with S protein

191 antigens from HCoV (HKU1, NL63, 229E), but not for SARS-CoV-2 (Supplementary Figure 1).

192 As shown in Figure 1A, all serum samples from PCR-confirmed COVID-19 patients collected

$193 \geq 7$ days from symptom onset had IgG above the determined cut-off in the RBD screening ELISA

$194(P<0.0001)$, while the pooled IVIG preparations (representing $>1,500$ donors each) were

195 negative.

196

197 To illustrate the utility of the second confirmatory ELISA, samples with absorbance close to the 198 cut-off in the RBD screen were titrated against the S protein. As shown in Figure 1B, the anti-S 199 protein IgG titration clearly separated the true positives from the false positives, as only the 200 PCR-confirmed COVID-19 samples met the seropositive criteria (OD > cut-off in at least two 201 consecutive dilutions). Indeed, this second ELISA resulted in all pre-pandemic samples being 202 classified as negative and a calculated specificity of $100 \%$, albeit in a moderately sized sample 203 panel. The use of IgM and IgA in the two-step ELISA protocol were also explored, however IgM 204 was found to have lower sensitivity with only 4/18 of the 7+ day COVID-19 samples being 205 seropositive, compared with 18/18 (100\%) for IgG. IgA was deemed unsuitable as the assay

206

207 208

209

210

211 window was inferior to that of IgG (OD range 0.01-0.82 compared with 0.01-1.42), and recent reports highlighted reduced sensitivity and specificity for IgA based SARS-CoV-2 ELISA (Meyer et al., 2020; Beavis et al., 2020).

Isotyping ELISA performed with the PCR-confirmed COVID-19 samples showed significantly higher titres for $\operatorname{IgG}$ compared with $\operatorname{IgM}$ and $\operatorname{IgA}$ for both the RBD and $\mathrm{S}$ protein (Figure $1 \mathrm{C}$ and $\mathrm{D}, P<0.01)$. Of note, only one sample had IgM levels higher than $\mathrm{IgG}$, with the remaining 20 
212 samples having higher IgG than IgM, despite $40 \%$ of the samples being collected within two-

213 weeks of symptom onset.

214

215

\section{Neutralising anti-SARS-CoV-2 antibodies}

216 The presence of neutralising antibodies (NAbs) capable of blocking the interaction between the 217 SARS-CoV-2 RBD and the hACE-2 receptor was assessed using sVNT (Figure 2A) (Tan et al., 218 2020). To validate performance, the pre-pandemic panel $(n=113)$ was compared with the PCR219 confirmed COVID-19 samples. Using the cut-off value of $20 \%$ inhibition recommended by the 220 manufacturer, all control samples tested were negative, resulting in a 100\% specificity. Similarly, 221 all sera from PCR-confirmed COVID-19 patients collected $\geq 7$ days from symptom onset were 222 positive for a sensitivity of $100 \%$. An experimentally calculated cut-off of $19.59 \%$ (mean +3 SD 223 of controls) also gave 100\% sensitivity and there was a significant increase in the level of NAbs 224 (\% inhibition) in the 7+ day COVID-19 samples compared with controls $(P<0.001)$.

225 A correlation analysis of the sVNT with ELISA titre data for IgG, IgM and IgA against the RBD 226 and S protein found the highest correlation between the sVNT and anti-RBD IgG (r 0.91, $P<$ 227 0.0001), suggesting anti-RBD IgG is the best predictor of neutralisation (Figure 2B). Temporal 228 samples were available for three of the PCR-confirmed COVID-19 patients, with increasing 229 NAbs detected in patient one between days 6 and 9, and higher levels of NAbs in patients two 230 and three between days 11 and 31 and days 15 and 31, respectively (Figure 2C). In keeping, 231 there was a significant, positive correlation between days from symptom onset and the level of 232 NAbs (\% inhibition) in the PCR-confirmed COVID-19 patient group up to 40 days (Figure 2D; r $2330.54, P<0.05)$.

Patients with COVID-19-like symptoms

235 To assess the utility of the two-step ELISA protocol and the sVNT in detecting prior 236 SARS-CoV-2 infection, 82 patients who presented to hospital during the lockdown period with 237 respiratory symptoms fitting the case definition for COVID-19 testing, but negative for PCR, 238 were analysed. A single patient was classified as seropositive in the two-step ELISA (RBD 239 screen, OD 0.87; S protein titration OD $>0.2$ in four consecutive dilution wells, Supplementary 240 data). Similarly, only one patient was classified as positive in the sVNT with $65.5 \%$ inhibition 
241 (Figure 2A). This was the same patient identified using the two-step ELISA, indicating a prior 242 undetected SARS-CoV-2 infection. Although the patient was PCR negative at presentation, they

243 had notable travel history as a risk factor.

\section{DISCUSSION}

245 At the time of writing, New Zealand has eliminated community transmission of SARS-CoV-2.

246 This is a very different scenario to that during Alert Level 4 Lockdown, when human activity

247 was severely restricted, and the perceived need for rapid establishment of serological assays was

248 significant. Through the generous sharing of reagents and technical expertise by the international

249 scientific community, and highly collaborative efforts of scientists and clinicians across the

250 country we were able to establish and assess the described serological assays in a matter of

251 weeks. These assays detect SARS-CoV-2 IgG and the presence of neutralising antibodies, in

252 persons who have been infected with SARS-CoV-2 at least 7 days after symptom onset, with

253 repeat sampling recommended in those where samples are obtained $<7$ days from symptom

254 onset. While the lack of community transmission in New Zealand has limited the scale of

255 serological investigations thus far, maintaining our current state requires near-perfect

256 management of returning travellers, as new cases are imported. Serological assays could have a

257 key role in our border setting, identifying persons who have previously had a SARS-CoV-2

258 infection. Furthermore, the application of serological assays that enable accurate measurement

259 and further understanding of SARS-CoV-2 immune responses are crucial to any "exit strategy"

260 reliant on effective vaccines and/or therapeutics (Baker, Kvalsvig \& Verrall, 2020).

261 The two-step ELISA protocol is based on published protocols (Stadlbauer et al., 2020), with the

262 seropositive cut-off established using a panel of pre-pandemic sera that showed bacterial

263 pneumonia and other common respiratory infections such as rhinovirus, influenza and respiratory

264 syncytial virus did not cross-react with the SARS-CoV-2 RBD and S proteins. Although the pre-

265 pandemic panel does not include samples from known human coronavirus infections, the healthy

266 control sera in the panel had broad reactivity with $\mathrm{HCoV}$ antigens. This is consistent with other

267 studies have reported that the majority of banked pre-pandemic sera react with human

268 coronavirus antigens given the ubiquitous nature of these infections (Amanat et al., 2020; Juno et

269 al., 2020). Furthermore, neither of the pooled IVIG preparations derived from $>1500$ donors 
270 tested in this study reacted with SARS-CoV-2 RBD or S proteins, consistent with the negligible

271 cross-reactivity of human coronavirus sera reported by the assay developers (Amanat et al.,

272 2020; Tan et al., 2020).

273 All PCR-confirmed COVID-19 patients showed strong seroconversion a week after symptom 274 onset, with mean ELISA AUC titre for anti-RBD IgG of 1:1,500. Although our sample size was

275 limited, the antibody responses follow trends observed in larger COVID-19 cohorts in settings

276 with higher case numbers. This includes a concurrent rise in IgM and IgG (Huang et al., 2020;

277 To et al., 2020), and robust antibody responses to the full-length S protein, as well as RBD (Chen

278 et al., 2020; Amanat et al., 2020; Juno et al., 2020). The level of NAbs, which block the

279 interaction of the RBD with the hACE-2 receptor was highly correlated with anti-RBD IgG in

280 this study. This is in keeping with observations that most SARS-CoV-2 neutralising epitopes are

281 localised in the RBD (Ju et al., 2020; Chen et al., 2020) and that neutralisation measured by

282 conventional, viral neutralisation assays is reported to correlate with the sVNT (Tan et al., 2020).

283 While more extensive comparison of the sVNT with gold standard viral neutralisation assays is

284 required, the ability of the assay to measure total NAbs in less than 2 hours, without the need to

285 handle live SARS-CoV-2 virus, provides strong rationale to consider these types of assays in

286 pandemic management, particularly in settings where BSL3 laboratory infrastructure is limited.

287 Serological tests are traditionally used to support clinical diagnosis by determining recent or prior 288 infection when swab results are negative (Bryant et al., 2020). In the context of SARS-CoV-2 infections, serology could confirm diagnosis in individuals who are PCR-negative due to late presentation or technical limitations of swab-based tests. Proof of principle was demonstrated in this study by the identification of a seropositive individual in the two-step ELISA and the sVNT from a cohort of 82 patients with respiratory symptoms that presented to hospital during the Alert level 4 lockdown period. The positive serology results, combined with travel history, suggest a prior undetected SARS-CoV-2 infection and highlight the value of accurate serology in clinical diagnosis. Extending the application of serology to understand SARS-CoV-2 transmission within clusters was recently demonstrated in Singapore, where detection of seropositive individuals enabled three small clusters to be connected (Yong et al., 2020). Beyond transmission studies, serological testing in managed isolation facilities could provide a more complete picture of 
299 previous SARS-CoV-2 infections in returning citizens in settings like New Zealand where strict 300 border restrictions remain.

301 Large scale serosurveys require careful consideration of assay sensitivity and specificity. While

302 the serological assays described in this study show very high sensitivity and specificity,

303 markedly larger cohorts would need to be tested before robust assessments of accuracy can be

304 performed. Indeed, the need for rigorously validated assays is arguably greater in low prevalence

305 settings like New Zealand before wide-spread serosurveys are considered. Even an assay with

306 near perfect specificity of 99.9\% would identify 100 false positives in every 100,000 individuals,

307 and with $<0.1 \%$ of the New Zealand population having been infected, positive predictive value is

308 extremely limited. In contrast, targeted studies of high-risk individuals such as health care

309 workers, those linked with clusters and in managed isolation would provide an opportunity to

310 assess serological assay performance, and generate critical local data on levels of antibodies in

311 those with symptomatic versus asymptomatic infection (Krammer \& Simon, 2020; Bryant et al.,

312 2020). Studies that incorporate RBD-based assay such as those described here, as well as the

313 nucleoprotein as per the high-throughput systems recently launched by Roche and Abbott (Bryan

314 et al., 2020), would provide insight into SARS-CoV-2 exposure together with antibody

315 neutralisation, and lay the foundation for future studies aimed at understanding long-term

316 antibody persistence and vaccine efficacy.

\section{CONCLUSION}

318 In summary, the collective support of international colleagues combined with a collaborative 319 domestic network of scientists and clinicians enabled serological assays for COVID-19 to be 320 established during a nationwide lockdown. The success of the open approach we have taken may 321 offer considerable advantages in other settings where access to reagents and resources is

322 currently constrained. Importantly, these assays showed that hospitalised patients infected with

323 SARS-CoV-2 develop high levels of neutralising antibodies. While the low prevalence of

324 COVID-19 infections in New Zealand currently limits the use of serological assays in population

325 level serosurveys, they have immediate utility in clinical diagnostics, studies to understand

326 transmission in high-risk cohorts and underpinning longer-term "exit" strategies based on

327 effective vaccines and/or therapeutics. 
328

329

330

ACKNOWLEDGMENTS

331 We thank Shirley Lawrence and Franklin Han for assistance with patient recruitment, and Paul

332 Austin for laboratory support. Florian Krammer is thanked for the timely provision of vectors for 333 expression of the proteins used in this study. We are grateful to Linfa Wang at Duke-NUS and 334 GenScript for providing sVNT testing kits and technical advice.

335

336

337 
338

339

340

341

342

343

344

345

346

347

348

349

350

351

352

353

354

355

356

357

358

359

360

361

362

363

364

365

366

367

368

369

370

371

372

373

374

375

376

377

378

379

380

381

382

\section{REFERENCES}

Abbasi J 2020. The Promise and Peril of Antibody Testing for COVID-19. JAMA. DOI: 10.1001/jama.2020.6170.

Amanat F, Stadlbauer D, Strohmeier S, Nguyen THO, Chromikova V, McMahon M, Jiang K, Arunkumar GA, Jurczyszak D, Polanco J, Bermudez-Gonzalez M, Kleiner G, Aydillo T, Miorin L, Fierer DS, Lugo LA, Kojic EM, Stoever J, Liu STH, Cunningham-Rundles C, Felgner PL, Moran T, García-Sastre A, Caplivski D, Cheng AC, Kedzierska K, Vapalahti O, Hepojoki JM, Simon V, Krammer F 2020. A serological assay to detect SARS-CoV-2 seroconversion in humans. Nature medicine 5:562-12. DOI: 10.1038/s41591-020-0913-5. Anderson DE, Tan CW, Chia WN, Young BE, Linster M, Low JH, Tan Y-J, Chen MIC, Smith GJD, Leo YS, Lye DC, Wang L-F 2020. Lack of cross-neutralization by SARS patient sera towards SARS-CoV-2. Emerging microbes \& infections 9:900-902. DOI: $10.1080 / 22221751.2020 .1761267$.

Baker MG, Kvalsvig A, Verrall AJ 2020. New Zealand's COVID-19 elimination strategy. The Medical Journal of Australia.

Beavis KG, Matushek SM, Abeleda APF, Bethel C, Hunt C, Gillen S, Moran A, Tesic V 2020. Evaluation of the EUROIMMUN Anti-SARS-CoV-2 ELISA Assay for detection of IgA and IgG antibodies. Journal of clinical virology : the official publication of the Pan American Society for Clinical Virology 129:104468. DOI: 10.1016/j.jcv.2020.104468.

Bennett J, Moreland NJ, Oliver J, Crane J, Williamson DA, Sika-Paotonu D, Harwood M, Upton A, Smith S, Carapetis J, Baker MG 2019. Understanding group A streptococcal pharyngitis and skin infections as causes of rheumatic fever: protocol for a prospective disease incidence study. BMC infectious diseases 19:633. DOI: 10.1186/s12879-019-4126-9.

Bryan A, Pepper G, Wener MH, Fink SL, Morishima C, Chaudhary A, Jerome KR, Mathias PC, Greninger AL 2020. Performance Characteristics of the Abbott Architect SARS-CoV-2 IgG Assay and Seroprevalence in Boise, Idaho. Journal of clinical microbiology. DOI: 10.1128/JCM.00941-20.

Bryant JE, Azman AS, Ferrari MJ, Arnold BF, Boni MF, Boum Y, Hayford K, Luquero FJ, Mina MJ, Rodriguez-Barraquer I, Wu JT, Wade D, Vernet G, Leung DT 2020. Serology for SARS-CoV-2: Apprehensions, opportunities, and the path forward. Science immunology 5:eabc6347. DOI: 10.1126/sciimmunol.abc6347.

Chen X, Li R, Pan Z, Qian C, Yang Y, You R, Zhao J, Liu P, Gao L, Li Z, Huang Q, Xu L, Tang J, Tian Q, Yao W, Hu L, Yan X, Zhou X, Wu Y, Deng K, Zhang Z, Qian Z, Chen Y, Ye L 2020. Human monoclonal antibodies block the binding of SARS-CoV-2 spike protein to angiotensin converting enzyme 2 receptor. Cellular \& molecular immunology 579:265. DOI: $10.1038 / \mathrm{s} 41423-020-0426-7$.

Diamond MS, Pierson TC 2020. The Challenges of Vaccine Development against a New Virus during a Pandemic. Cell host \& microbe 27:699-703. DOI: 10.1016/j.chom.2020.04.021.

Duan K, Liu B, Li C, Zhang H, Yu T, Qu J, Zhou M, Chen L, Meng S, Hu Y, Peng C, Yuan M, Huang J, Wang Z, Yu J, Gao X, Wang D, Yu X, Li L, Zhang J, Wu X, Li B, Xu Y, Chen W, Peng Y, Hu Y, Lin L, Liu X, Huang S, Zhou Z, Zhang L, Wang Y, Zhang Z, Deng K, Xia Z, Gong Q, Zhang W, Zheng X, Liu Y, Yang H, Zhou D, Yu D, Hou J, Shi Z, Chen S, Chen Z, Zhang X, Yang X 2020. Effectiveness of convalescent plasma therapy in severe COVID-19 patients. Proceedings of the National Academy of Sciences of the United States of America. DOI: $10.1073 /$ pnas.2004168117. 
383

384

385

386

387

388

389

390

391

392

393

394

395

396

397

398

399

400

401

402

403

404

405

406

407

408

409

410

411

412

413

414

415

416

417

418

419

420

421

422

423

424

425

426

427

Garcia-Basteiro AL, Moncunill G, Tortajada M, Vidal M, Guinovart C, Jimenez A, Santano R, Sanz S, Mendez S, Llupia A, Aguilar R, Alonso S, Barrios D, Carolis C, Cistero P, Choliz E, Cruz A, Fochs S, Jairoce C, Hecht J, Lamoglia M, Martinez MJ, Mitchell R, Ortega N, Pey N, Puyol L, Ribes M, Rosell N, Sotomayor P, Torres S, Williams S, Barroso S, Vilella A, Munoz J, Varela P, Trilla A, Mayor A, Dobano C 2020. Seroprevalence of antibodies against SARS-CoV-2 among health care workers in a large Spanish reference hospital. MedRxix:136. DOI: 10.1101/2020.04.27.20082289.

Huang AT, Garcia-Carreras B, Hitchings MDT, Yang B, Katzelnick L, Rattigan SM, Borgert B, Moreno C, Solomon BD, Rodriguez-Barraquer I, Lessler J, Salje H, Burke DS, Wesolowski A, Cummings DAT 2020. A systematic review of antibody mediated immunity to coronaviruses: antibody kinetics, correlates of protection, and association of antibody responses with severity of disease. MedRxix:1-47. DOI: 10.1101/2020.04.14.20065771.

Ju B, Zhang Q, Ge X, Wang R, Yu J, Shan S, Zhou B, Song S, Tang X, Yu J, Ge J, Lan J, Yuan

J, Wang H, Zhao J, Zhang S, Wang Y, Shi X, Liu L, Wang X, Zhang Z, Zhang L 2020.

Potent human neutralizing antibodies elicited by SARS-CoV-2 infection. bioRxiv:1-42.

DOI: $10.1101 / 2020.03 .21 .990770$.

Juno JA, Tan H-X, Lee WS, Reynaldi A, Kelly HG, Wragg K, Esterbauer R, Kent HE, Batten CJ, Mordant FL, Gherardin NA, Pymm P, Dietrich MH, Scott NE, Tham W-H, Godfrey DI, Subbarao K, Davenport MP, Kent SJ, Wheatley AK 2020. Humoral and circulating follicular helper $\mathrm{T}$ cell responses in recovered patients with COVID-19. Nature medicine 52:583. DOI: 10.1038/s41591-020-0995-0.

Krammer F, Simon V 2020. Serology assays to manage COVID-19. Science (New York, NY). DOI: $10.1126 /$ science.abc1227.

Long Q-X, Liu B-Z, Deng H-J, Wu G-C, Deng K, Chen Y-K, Liao P, Qiu J-F, Lin Y, Cai X-F, Wang D-Q, Hu Y, Ren J-H, Tang N, Xu Y-Y, Yu L-H, Mo Z, Gong F, Zhang X-L, Tian WG, Hu L, Zhang X-X, Xiang J-L, Du H-X, Liu H-W, Lang C-H, Luo X-H, Wu S-B, Cui X-P, Zhou Z, Zhu M-M, Wang J, Xue C-J, Li X-F, Wang L, Li Z-J, Wang K, Niu C-C, Yang Q-J, Tang X-J, Zhang Y, Liu X-M, Li J-J, Zhang D-C, Zhang F, Liu P, Yuan J, Li Q, Hu J-L, Chen J, Huang A-L 2020. Antibody responses to SARS-CoV-2 in patients with COVID-19. Nature medicine 62:477-4. DOI: 10.1038/s41591-020-0897-1.

Meyer B, Torriani G, Yerly S, Mazza L, Calame A, Arm-Vernez I, Zimmer G, Agoritsas T, Stirnemann J, Spechbach H, Guessous I, Stringhini S, Pugin J, Roux-Lombard P, Fontao L, Siegrist CA, Eckerle I, Vuilleumier N, Kaiser L, Geneva Center for Emerging Viral Diseases 2020. Validation of a commercially available SARS-CoV-2 serological immunoassay. Clinical microbiology and infection : the official publication of the European Society of Clinical Microbiology and Infectious Diseases. DOI: 10.1016/j.cmi.2020.06.024.

Petherick A 2020. Developing antibody tests for SARS-CoV-2. The Lancet 395:1101-1102. DOI: $10.1016 / \mathrm{S} 0140-6736(20) 30788-1$.

Sethuraman N, Jeremiah SS, Ryo A 2020. Interpreting Diagnostic Tests for SARS-CoV-2. $J A M A$. DOI: 10.1001/jama.2020.8259.

Stadlbauer D, Amanat F, Chromikova V, Jiang K, Strohmeier S, Arunkumar GA, Tan J, Bhavsar D, Capuano C, Kirkpatrick E, Meade P, Brito RN, Teo C, McMahon M, Simon V, Krammer F 2020. SARS-CoV-2 Seroconversion in Humans: A Detailed Protocol for a Serological Assay, Antigen Production, and Test Setup. Current protocols in microbiology 57:e100. DOI: $10.1002 /$ cpmc.100. 
428 Tan CW, Chia WN, Qin X, Liu P, Chen MIC, Tiu C, Hu Z, Chen VC-W, Young BE, Sia WR,

429

430

431

432

433

434

435

436

437

438

439

440

441

442

443 Tan Y-J, Foo R, Yi Y, Lye DC, Anderson DE, Wang L-F 2020. A SARS-CoV-2 surrogate virus neutralization test based on antibody-mediated blockage of ACE2-spike protein-protein interaction. Nature biotechnology 395:470. DOI: 10.1038/s41587-020-0631-z.

To KK-W, Tsang OT-Y, Leung W-S, Tam AR, Wu T-C, Lung DC, Yip CC-Y, Cai J-P, Chan JM-C, Chik TS-H, Lau DP-L, Choi CY-C, Chen L-L, Chan W-M, Chan K-H, Ip JD, Ng ACK, Poon RW-S, Luo C-T, Cheng VC-C, Chan JF-W, Hung IF-N, Chen Z, Chen H, Yuen K$\mathrm{Y}$ 2020. Temporal profiles of viral load in posterior oropharyngeal saliva samples and serum antibody responses during infection by SARS-CoV-2: an observational cohort study. The Lancet Infectious Diseases 20:565-574. DOI: 10.1016/S1473-3099(20)30196-1.

Yong SEF, Anderson DE, Wei WE, Pang J, Chia WN, Tan CW, Teoh YL, Rajendram P, Toh MPHS, Poh C, Koh VTJ, Lum J, Suhaimi N-AM, Chia PY, Chen MI-C, Vasoo S, Ong B, Leo YS, Wang L, Lee VJM 2020. Connecting clusters of COVID-19: an epidemiological and serological investigation. The Lancet Infectious Diseases 20:809-815. DOI: 10.1016/S1473-3099(20)30273-5. 


\section{FIGURE LEGENDS}

445 Figure 1. Antibody responses in pre-pandemic controls and PCR confirmed COVID-19 sera. A,

446 Screening IgG ELISA against RBD for pre-pandemic controls (orange), IVIG (pink squares) and

447 PCR confirmed COVID-19 sera $<7$ (light blue) and 7+ days from symptom onset (royal blue).

448 Samples boxed in grey above the cut-off (red dashed line) were titrated against the Spike protein.

449 B, Example of the confirmatory IgG titration ELISA against Spike protein. Samples above the

450 cut-off (dashed line) in at least two consecutive dilutions are deemed seropositive (royal blue).

451 Isotype specific ELISA against the RBD (C) and Spike (D) for IgG (blue), IgM (green) and IgA

452 (red) in PCR confirmed COVID-19 sera $<7$ and 7+ days from symptom onset. One patient

453 showed higher IgM than IgG responses and is indicated (\#). AUC = Area under the curve.

454 Illustrations created with BioRender.com

455 Figure 2. Surrogate viral neutralisation test (sVNT). A, Negative controls (orange) and PCR 456 confirmed COVID-19 sera $<7$ (light blue) and 7+ days from symptom onset (royal blue).

457 Samples with inhibition above the cut-off (red dashed line, 20\%) were deemed positive.

458 Following validation, the sVNT was run on PCR negative (ND) samples with COVID-like

459 symptoms (grey). One sample showed 65.5\% inhibition (green) indicating positive SARS-CoV-2

460 neutralisation. B, Pearson correlation for \% inhibition (sVNT) and IgG antibody titre to RBD in

461 PCR confirmed COVID-19 sera ( $\mathrm{n}=21)$. Inset, Pearson correlation coefficients comparing \%

462 inhibition (sVNT) and antibody isotype (IgG, IgA and $\operatorname{IgM}$ responses) to RBD (left) and Spike

463 protein (right). Colour scale from weak correlation (Pearsons coefficient of 0, white) to strong

464 correlation (Pearson's coefficient of 1, red). C, \% inhibition (sVNT) for temporal samples from 3

465 patients with PCR confirmed COVID-19 infection. D, Pearson correlation for \% inhibition

466 (sVNT) and days since symptom onset for PCR confirmed COVID-19 sera ( $\mathrm{n}=21)$. AUC = Area

467 under the curve, ND = not detected 


\section{Figure 1}

Figure 1. Antibody responses in pre-pandemic controls and PCR confirmed COVID-19 sera.

A, Screening IgG ELISA against RBD for pre-pandemic controls (orange), IVIG (pink squares) and PCR confirmed COVID-19 sera $<7$ (light blue) and 7+ days from symptom onset (royal blue). Samples boxed in grey above the cut-off (red dashed line) were titrated against the Spike protein. B, Example of the confirmatory IgG titration ELISA against Spike protein. Samples above the cut-off (dashed line) in at least two consecutive dilutions are deemed seropositive (royal blue). Isotype specific ELISA against the RBD (C) and Spike (D) for IgG (blue), IgM (green) and IgA (red) in PCR confirmed COVID-19 sera $<7$ and $7+$ days from symptom onset. One patient showed higher IgM than IgG responses and is indicated (\#). AUC $=$ Area under the curve. Illustrations created with BioRender.com 
A
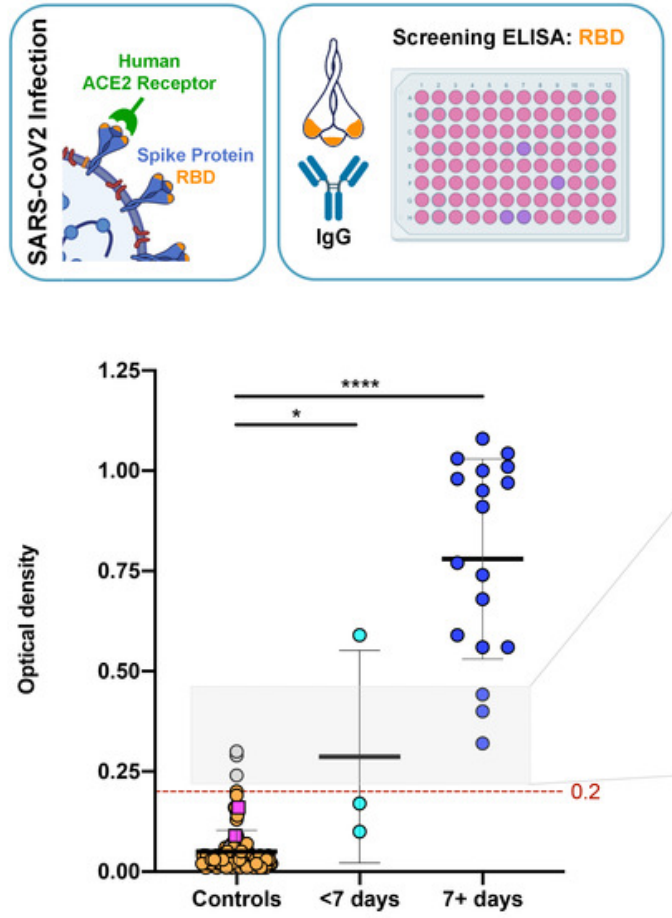

C

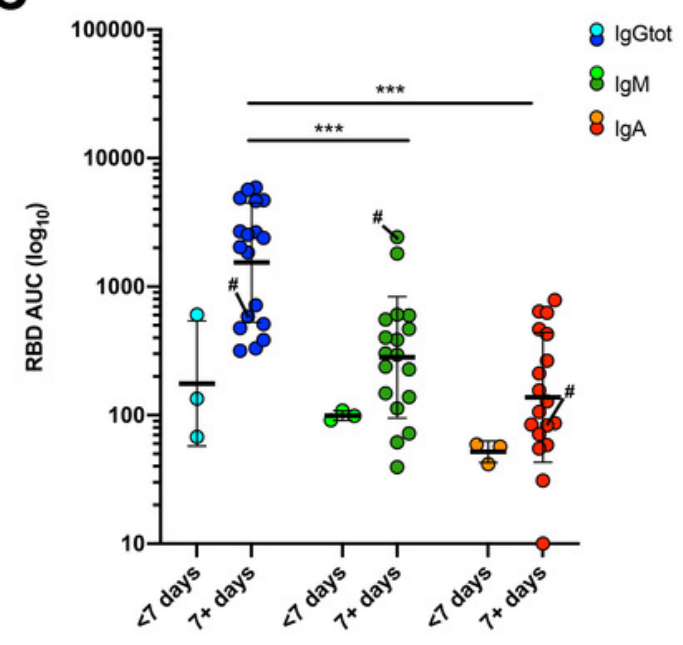

B
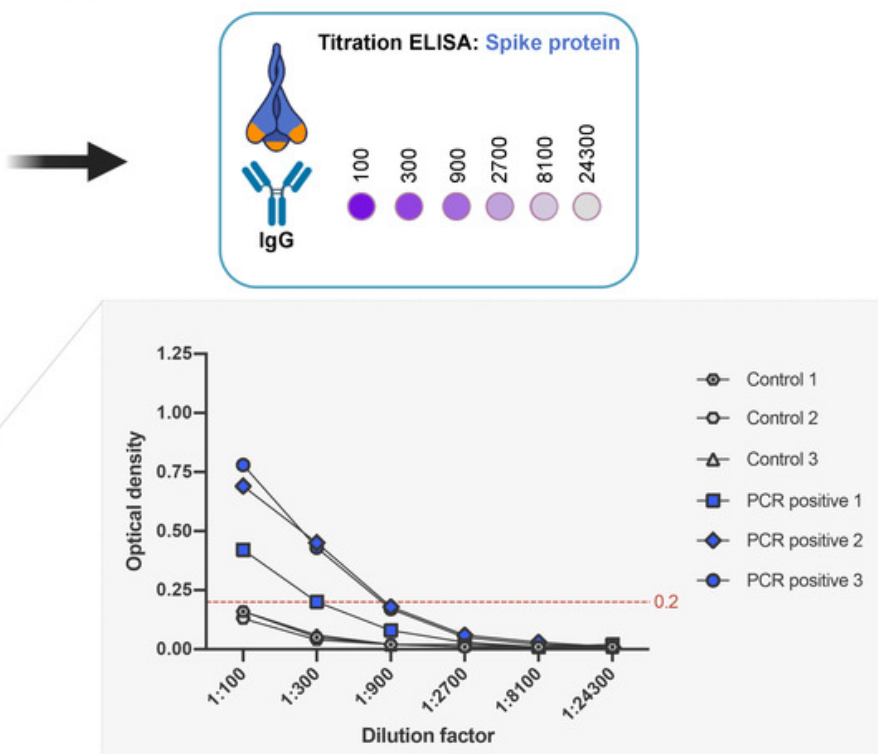

D

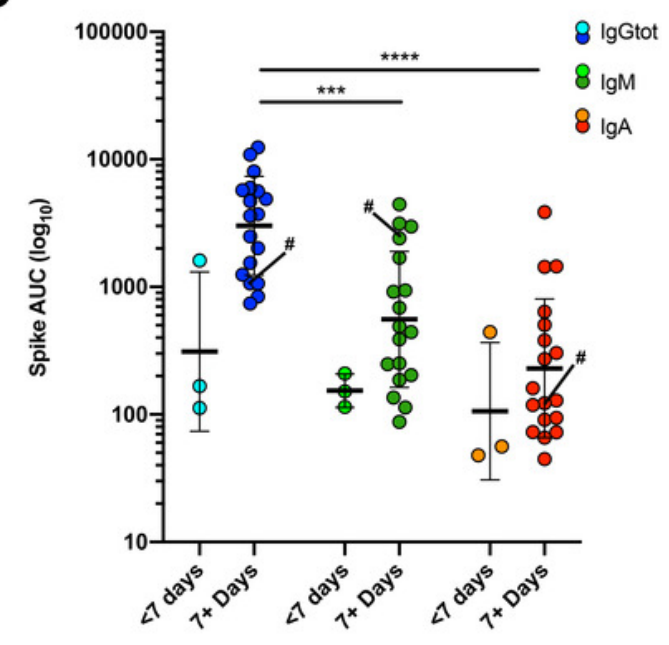




\section{Figure 2}

Figure 2. Surrogate viral neutralisation test (sVNT).

A, Negative controls (orange) and PCR confirmed COVID-19 sera $<7$ (light blue) and 7+ days from symptom onset (royal blue). Samples with inhibition above the cut-off (red dashed line, $20 \%$ ) were deemed positive. Following validation, the sVNT was run on PCR negative (ND) samples with COVID-like symptoms (grey). One sample showed 65.5\% inhibition (green) indicating positive SARS-CoV-2 neutralisation. B, Pearson correlation for $\%$ inhibition (sVNT) and IgG antibody titre to RBD in PCR confirmed COVID-19 sera $(n=21)$. Inset, Pearson correlation coefficients comparing \% inhibition (sVNT) and antibody isotype (IgG, IgA and IgM responses) to RBD (left) and Spike protein (right). Colour scale from weak correlation (Pearsons coefficient of 0 , white) to strong correlation (Pearson's coefficient of 1, red). C, \% inhibition (sVNT) for temporal samples from 3 patients with PCR confirmed COVID-19 infection. D, Pearson correlation for \% inhibition (sVNT) and days since symptom onset for PCR confirmed COVID-19 sera $(n=21)$. AUC $=$ Area under the curve, ND = not detected 
A

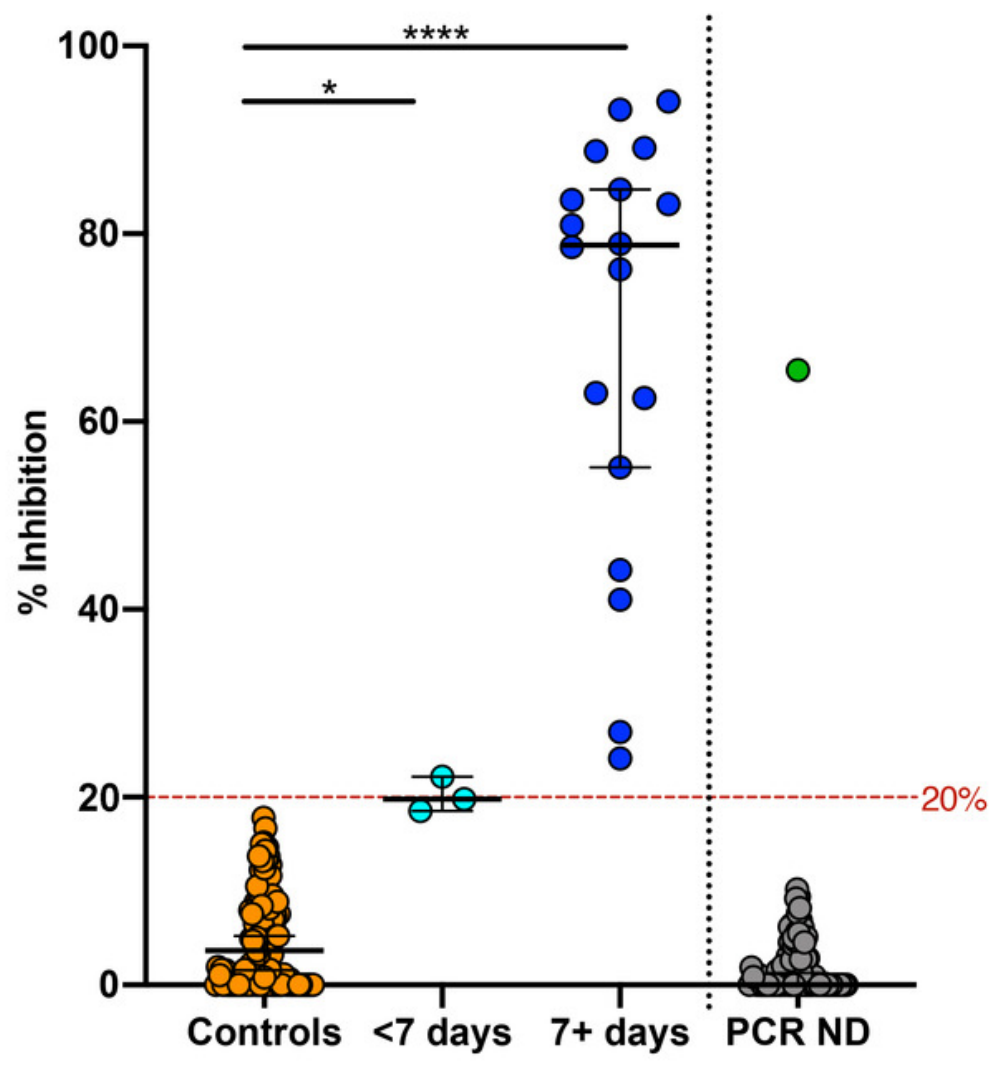

B

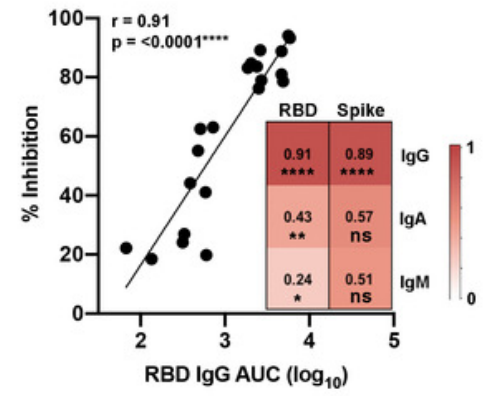

C

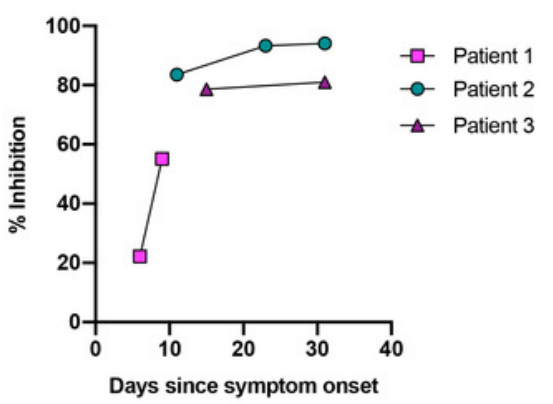

D

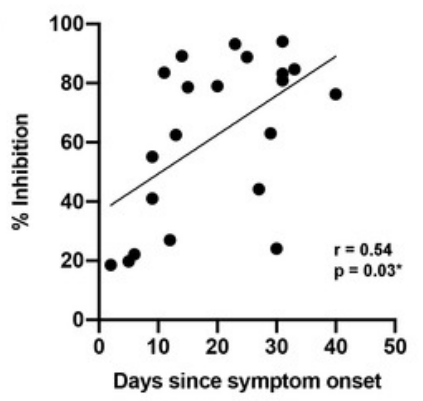




\section{Table $\mathbf{1}$ (on next page)}

Table 1. Patient demographics.

The pre-pandemic panel comprised paediatric respiratory infections, adults hospitalised with bacteraemia or bacterial pneumonia and healthy adult laboratory donors. The pandemic samples comprised PCR-confirmed COVID-19 and symptomatic PCR-negative groups. 
1 Table 1. Patient demographics. The pre-pandemic panel comprised paediatric respiratory infections, adults

2 hospitalised with bacteraemia or bacterial pneumonia and healthy adult laboratory donors. The pandemic samples

3 comprised PCR-confirmed COVID-19 and symptomatic PCR-negative groups.

4

\begin{tabular}{|c|c|c|c|c|c|c|}
\hline & & $\begin{array}{l}\text { Healthy } \\
\text { donors }\end{array}$ & $\begin{array}{l}\text { Respiratory } \\
\text { infections }\end{array}$ & $\begin{array}{l}\text { Hospitalised } \\
\text { infections }\end{array}$ & $\begin{array}{l}\text { COVID-19 } \\
\text { cases }\end{array}$ & $\begin{array}{l}\text { Symptomatic } \\
\text { PCR-negative }\end{array}$ \\
\hline \multicolumn{2}{|l|}{ Total } & 31 & 57 & 25 & 17 & 82 \\
\hline \multirow[t]{2}{*}{ Age } & Median & \multirow{2}{*}{$>20$ years } & 10 & 67 & 48 & 60 \\
\hline & Range & & $5-14$ & $19-93$ & $23-86$ & $17-94$ \\
\hline Gender & $\mathrm{M} / \mathrm{F}$ & $\mathrm{n} / \mathrm{a}^{*}$ & $30 / 27$ & $14 / 11$ & $7 / 10$ & $43 / 39$ \\
\hline Year collected & & 2014-2019 & $2018-2019$ & 2018 & 2020 & 2020 \\
\hline \multirow[t]{5}{*}{ Viral infections } & RSV & & 6 & & & \\
\hline & Influenza A & & 14 & & & \\
\hline & Influenza B & & 16 & & & \\
\hline & Parainfluenza 1 & & 1 & & & \\
\hline & Rhino/Enterovirus & & 7 & & & \\
\hline \multirow[t]{5}{*}{ Other infections } & Bacterial pharyngitis & & 2 & & & \\
\hline & Pertussis & & 4 & & & \\
\hline & Mycoplasma & & 7 & & & \\
\hline & Bacterial pneumonia & & & 5 & & \\
\hline & Bacteraemia & & & 20 & & \\
\hline
\end{tabular}

$5 \quad$ "not available

6

7 